\title{
REFLEXIVE OPEN MAPPINGS ON GENERALIZED GRAPHS
}

\author{
STANISLAW MIKLOS
}

(Received 16 September 1987)

Communicated by J. H. Rubinstein

\begin{abstract}
In this paper we show that a locally connected and locally compact metric image of a generalized graph under a reflexive open mapping is a generalized graph; further, we characterize all acyclic generalized graphs $X$ with the property that any locally one-to-one reflexive open mapping of $X$ into a Hausdorff space is globally one-to-one. Several problems are posed and some examples are given.
\end{abstract}

1980 Mathematics subject classification (Amer. Math. Soc.): primary $54 \mathrm{C} 10$; secondary $54 \mathrm{~F}$ 50,54 F 65.

Keywords and phrases: Hausdorff spaces, locally connected and locally compact metric spaces, generalized graph, reflexive open mappings, one-to-one mappings.

Whyburn [11, page 182] has shown that an open image of a graph (that is, a one-dimensional connected polytope) is a graph. Eberhart, Fugate and Gordh generalize this result, showing that the weakly confluent image of a graph is a graph [5, Theorem II.6]. In [1], we proved that the metric open image of a generalized graph (that is, a connected space embeddable into a graph) is a generalized graph, too. A generalization of open mappings is the class of reflexive open mappings. Recall that a (continuous) mapping $f$ from a topological space onto another topological space is said to be reflexive open if $f^{-1}(f(U))$ is open whenever $U$ is open (see [4, page 597]). Therefore the following problem is natural.

(C) 1989 Australian Mathematical Society $0263-6115 / 89 \$ A 2.00+0.00$ 
Problem 1. Characterize the class of all images of generalized graphs under reflexive open mappings.

An important subclass of reflexive open mappings are one-to-one mappings (see [3]). So we have

Problem 2. Characterize the class of all images of generalized graphs under one-to-one mappings.

In this paper we give a partial answer to Problems 1 and 2. Namely we reduce the class of images of generalized graphs under one-to-one mappings and also reflexive open mappings to locally connected and locally compact metric spaces. Moreover, we characterize all acyclic generalized graphs $X$ with the property that any locally one-to-one reflexive open mapping of $X$ into a Hausdorff space is globally one-to-one.

Lelek and McAuley [9, page 320] have proved the following

Theorem A (Lelek and McAuley). If a locally connected and locally compact metric space $Y$ is a one-to-one continuous image of the line, then $Y$ is homeomorphic to one of the five objects (I)-(V) listed in Figure 1.

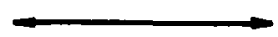

(I)

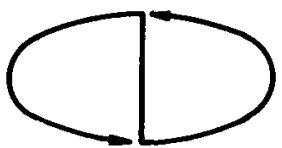

(IV)

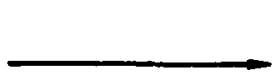

(VII)

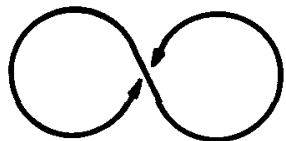

(II)

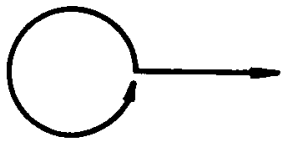

(V)

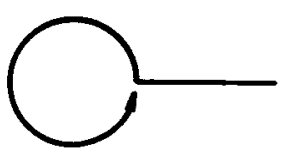

(VIII)

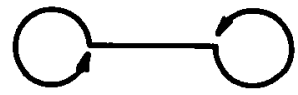

(III)

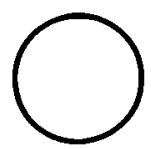

(VI)

(IX)

FIGURE 1

By an open arc we mean the homeomorphic image of the line and by a half-open arc we mean the homeomorphic image of the half-line. 
Applying these notions and using the same argument as in the proof of $[9$, Theorem 1] we get

THEOREM 1. If a locally connected and locally compact metric space $Y$ is a one-to-one continuous image of the half-open arc, then $Y$ is homeomorphic to one of the three objects (VI)-(VIII) of Figure 1.

Now we prove a proposition which will be used later to obtain a stronger result (Theorem 2 below).

Proposition. Let $X$ be a generalized graph, $Y$ a locally connected and locally compact metric space, and let $f$ be a one-to-one mapping from $X$ onto $Y$. Then $Y$ is a generalized graph.

Proof. If $X$ is either an open arc or a half-open arc, then Theorems $\mathrm{A}$ and 1 imply that $f(X)$ is a generalized graph. Thus let $X$ be an arbitrary generalized graph different from an open arc and a half-open arc. Further, if $X$ is a graph, then $f$ is a homeomorphism, whence $f(X)$ is a graph. If $X$ is not a graph (that is, if $X$ is not compact), then there exists a graph $G$ and a homeomorphic embedding $h: X \rightarrow G$ such that $G \backslash h(X)$ is a finite set of end points of $G$ (see [1, Theorem 1(iii)]). We put $G^{\prime}=h(X)$ and $G \backslash G^{\prime}=\left\{e_{1}, e_{2}, \ldots, e_{n}\right\}$. For $i=1,2, \ldots, n$, let $e_{i}^{\prime}$ denote a ramification point of $G^{\prime}$ adjacent to $e_{i}$, and let each $\operatorname{arc} e_{i}^{\prime} e_{i}$ be ordered from $e_{i}^{\prime}$ to $e_{i}$. Further, let $a_{1}^{i}, a_{2}^{i}, a_{3}^{i}, \ldots$ be an infinite increasing sequence tending to $e_{i}$, where $a_{1}^{i}=e_{i}^{\prime}$ and $a_{j}^{i} \in e_{i}^{\prime} e_{i} \backslash\left\{e_{i}\right\}$, whenever $j \in\{1,2,3, \ldots\}$. For $i=1,2, \ldots, n$ we consider the set $H^{i}=\bigcap_{j=1}^{\infty} \operatorname{cl}\left\{g(x): x \geq a_{j}^{i}\right\}$, where $g: G^{\prime} \rightarrow Y$ is defined by $g=f h^{-1}$. Since $Y$ is locally connected and locally compact, we conclude that $H^{i}$ is either empty or a singleton (compare with the proof of [9, Theorem 1]). Now, if $H^{i}=\varnothing$ for $i=1,2, \ldots, n$, then $g$ is a homeomorphism, whence $Y$ is a generalized graph. If we have $H^{i}=\{p\}$ for some $i \in\{1,2, \ldots, n\}$, then the mapping $g$ is not a homeomorphism because the function $g^{-1}: Y \rightarrow G^{\prime}$ is not continuous at the point $p$. Thus, if $q \in g^{-1}(p)$, then $\operatorname{ord}_{q} G^{\prime}<\operatorname{ord}_{p} Y$, where $\operatorname{ord}_{z} Z$ denotes the Menger-Urysohn order at a point $z$ in a space $Z$ (see [11, page 48] or [8, page 274]). The point $p$ is contained in the intersection of at most $n$ sets $H^{i}$. Thus, since $g$ is one-to-one we have $\operatorname{ord}_{p} Y \leq \operatorname{ord}_{q} G^{\prime}+n$. Further, since the number of the sets $H^{i}$ which are non-empty is finite and $G^{\prime}$ is a generalized graph, we have that (1) all points of $Y$ are of some finite order, and (2) almost all points of $Y$ are of order two. Since $Y$ is connected as a continuous image of a connected space, it must be a generalized continuum, that is, a connected and locally compact space. Hence by (1), (2) and $[1$, Theorem $1(v)] Y$ is a generalized graph. The proof is complete. 
The proposition can be generalized as follows.

THEOREM 2. Let $X$ be a generalized graph, $Y$ a locally connected and locally compact metric space, and let $f$ be a reflexive open mapping from $X$ onto $Y$. Then $Y$ is a generalized graph.

Proof. From [12, Theorem 5] (compare with [4, Theorem 3.6]), $f$ factors uniquely as $f=h m$ where $m: X \rightarrow m(X)$ is an open mapping and $h: m(X) \rightarrow Y$ is a one-to-one mapping. Since $X$ is a generalized graph and $Y$ is metric, $m(X)$ is also a generalized graph (see [1, Theorem 2]). Thus, by the proposition, $Y$ is a generalized graph and the proof is complete.

ReMarK 1. It is not difficult to check that local connectedness is an essential condition in all theorems above (compare a remark in [9, page 321]). For instance, the "Warsaw circle" (that is, the curve $\sin (1 / x)$ ) is a one-to-one and continuous image of the half-line. However, by [6, Theorem 2] we infer that local connectedness, in all theorems above, can be replaced by the condition that $Y$ is connected im kleinen or, more generally, by aposyndesis of $Y$. Further, by [6, Theorem 4] it can be seen that planability of $Y$ can be substituted for local compactness in all theorems above.

Jungck [7, page 43 ] has proved that if a locally connected and a locally compact metric space $Y$ is the image of the line under a reflexive open map which is either locally or globally one-to-one, then $Y$ is homeomorphic to one of the six objects (I)-(VI) above (Figure 1). From [12, Theorem 5] it is easy to show the following

THEOREM 3. If a locally connected and locally compact metric space $Y$ is the image of the line under a reflexive open mapping, then $Y$ is homeomorphic to one of the nine objects (I)-(IX) above (see Figure 1).

Let a graph $G$, containing at least one end point $e$, be given. A generalized graph $G \backslash\{e\}$ will be called a 1 -generalized graph.

Using this notion we formulate the next result.

THEOREM 4. Any locally one-to-one reflexive open mapping of an acyclic generalized graph $X$ into a Hausdorff space $Y$ is globally one-to-one if and only if $X$ is either a graph or a generalized graph.

Proof. First we prove that, if an acyclic generalized graph $X$ is a graph or a 1-generalized graph, then any locally one-to-one reflexive open mapping $f$ from $X$ into a Hausdorff space $Y$ is globally one-to-one. Indeed, if $X$ is 
a graph, then $f$ is open by [4, Corollary 3.7.2], whence it is a local homeomorphism (for the definition see [11, page 199]) on $X$. Acyclicity of $X$ implies that $f$ is a homeomorphism (see [2, Corollary 2]). If $X$ is an acyclic 1-generalized graph we suppose on the contrary that there is some $f$ which is locally one-to-one but not globally one-to-one. Thus there are two different points $a, b$ in $X$ with $f(a)=f(b)$. Since an arc $a b$ is compact and $f$ is locally one-to-one, there exists an arc $c d$ in $a b$ such that $f(c)=f(d)$ and $f$ is globally one-to-one on the half-open arc $c d \backslash\{d\}$. But then $f(c d)$ is homeomorphic to a simple closed curve $S$. We consider $f^{-1}(S)$. Since $S$ is compact in the Hausdorff space $Y$, it is closed. Thus $f^{-1}(S)$ is closed in $X$. Let $C$ be a component of $f^{-1}(S)$ containing the arc $c d$. Note that $C$ is atroidic. Otherwise, $C$ contains a ramification point $r$. Since $f$ is locally one-to-one, there is a closed neighbourhood $U$ of $r$ such that the partial mapping $f \mid U: U \rightarrow S$ is globally one-to-one. Since $U$ contains a ramification point, also $S$ contains a ramificiation point, a contradiction. Further, since $X$ is a 1-generalized graph, $C$ is either an arc or a half-open arc, and hence $C$ contains at least one end point $e$ (of $C$ ). Let $V$ be an open neighborhood of $e$ such that the partial mapping $f \mid \bar{V}$ is one-to-one. Further, consider a component $K$ of $f^{-1}(f(V))$ such that $K \cap V=\varnothing$ and $K \cap c d \neq \varnothing$. Note that if $f(e)=f(c)=f(d)$, then $K \cap c d=\{p\}$, where $p \in f^{-1}(f(e))$, and if $f(e) \neq f(c)=f(d)$, then $K \cap c d$ is either an arc or a half-open arc with an end point $q \in f^{-1}(f(e))$. Thus $K \cap c d$ is not open in $c d$, and moreover we conclude $K$ is not open in $X$. Hence $f^{-1}(f(V))$ is not open in $X$, contrary to reflexive openness of $f$. This contradiction shows that $f$ is globally one-to-one.

Second, let $X$ be an acyclic generalized graph which is neither a graph nor a 1-generalized graph. We show that there exists a locally one-to-one reflexive open mapping from $X$ into a Hausdorff space $Y$ which is not globally one-to-one. Indeed, since $X$ is neither a graph nor a 1-generalized graph, it contains an open arc $A$ which is closed in $X$ (see the definition of an open arc). Let $a b$ be an arc in $A$ whose interior contains all ramification points of $X$ contained in $A$, and let $B$ and $C$ be components of $A \backslash a b$. Define a mapping $f$ on $X$ in such a way that the partial mappings $f \mid X \backslash B$ and $f \mid X \backslash C$ are homeomorphisms and $f(A)$ is homeomorphic to a simple closed curve.

It is easy to verify that $Y=f(X)$ is a generalized graph and $f$ is open (thus reflexive open) and locally one-to-one because $f \mid X \backslash B$ and $f \mid X \backslash C$ are homeomorphisms. However, $f$ is not globally one-to-one because $f(A)$ is homeomorphic to the simple closed curve. The proof is complete.

As a consequence of Theorem 4 we get the following corollary. 
COROLlary [7, Theorem (9.4)]. Any locally one-to-one and reflexive open map $f$ of the half-line into a Hausdorff space is globally one-to-one.

REMARK 2. Acyclicity of the generalized graph $X$ is a necessary hypothesis in Theorem 4 even if we additionally assume that $X$ is a graph. Indeed, for $X$ and $Y$ being graphs as in Figure 2, it is easy to define a locally one-toone reflexive open mapping from $X$ onto $Y$ which is not globally one-to-one. However any locally one-to-one reflexive open mapping on a graph is a local homeomorphism. Graphs admitting a local homeomorphism which is not a homeomorphism are characterized in [10].
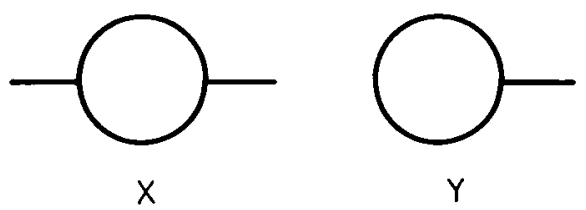

Figure 2

Problem 3. Characterize all non-compact generalized graphs $X$ with the property that any locally one-to-one and reflexive open mapping from $X$ into a Hausdorff space is globally one-to-one.

\section{References}

[1] J. J. Charatonik and S. Miklos, 'Generalized graphs and their open mappings', Rend. Mat. 2 (1982), 335-354.

[2] J. J. Charatonik and S. Miklos, 'A characterization of cyclic graphs', Bull. Acad. Polon. Sci. Sér. Sci. Math. 30 (1982), 453-455.

[3] E. Duda, 'One to one mappings and applications', General topology and its applications 1, pp. 135-142 (North-Holland Publishing Company, 1971).

[4] E. Duda and J. W. Smith, 'Reflexive open mappings', Pacific J. Math. 38 (1971), 597-611.

[5] C. A. Eberhart, J. B. Fugate, and G. R. Gordh, Jr., 'Branchpoint covering theorems for confluent and weakly confluent maps', Proc. Amer. Math. Soc. 55 (1976), 409-415.

[6] F. B. Jones, 'One-to-one continuous images of a line', General topology and its relations to modern analysis and algebra, Proceedings of the Kanpur Topological Conference, 1968, pp. $157-160$.

[7] G. F. Jungck, 'Local homeomorphisms', Dissertationes Math. (Rozprawy Mat.) 209 (1983).

[8] K. Kuratowski, Topology 2 (PWN, Warszawa, 1968).

[9] A. Lelek and L. F. McAuley, 'On hereditarily locally connected spaces and one-to-one continuous images of a line', Colloq. Math. 17 (1967), 319-324.

[10] S. Miklos, 'Local homeomorphisms onto cyclic graphs', Bull. Acad. Polon. Sci. Sér. Sci. Math. 31 (1983), 381-384. 
[11] G. T. Whyburn, Analytic topology (Amer. Math. Soc. Colloq. Publ., vol. 28, Providence, R.I., 1942).

[12] G. T. Whyburn, 'Open and closed mappings', Duke Math. J. 17 (1950), 69-74.

\section{Institute of Mathematics}

University of Wroclaw

pl. Grunwaldzki $2 / 4$

50-384 Wroclaw

Poland 